\title{
闭式泵控液气储能重载举升机构特性研究*
}

\author{
郝云晓 夏连鹏 权 龙 赵斌 程 珩 熊晓燕 \\ (太原理工大学新型传感器与智能控制教育部和山西省重点实验室 太原 030024)
}

\begin{abstract}
摘要: 采用变转速洜控缸闭式系统的重载举升机构, 在超越负载工况下, 若不采用制动电阻或能量回收装置, 系统将由于 电机不能支撑反向负载而失控。但制动电阻消耗电机的发电能量, 降低系统能效, 增加系统散热负担。针对上述问题, 提 出一种基于液气储能的重载举升机构闭式泵控三腔液压缸系统, 并将其应用于液压挖掘机动臂。液压洜与三腔液压缸的无 杆腔和有杆腔连接, 控制动臂运行; 配重腔与蓄能器连接平衡挖掘机执行机构的重量, 直接回收利用执行机构的势能。研 究中, 首先确定三腔液压缸和蓄能器的参数, 然后设计系统的控制策略, 最后构建闭式洜控三腔液压缸的挖掘机动臂试验 系统, 验证系统的可行性。试验结果表明, 合理设置蓄能器的压力, 不仅可以实现动臂势能的直接回收利用, 而且可以使 电机始终处于电动状态, 提高系统的可控性和能量效率, 与负载敏感控制的三腔液压缸系统相比, 液压泵输出能量降低 $27.2 \%$ 。
\end{abstract}

关键词: 液压挖掘机; 液气储能平衡; 三腔液压缸; 闭式原控系统; 能量回收利用 中图分类号: TH137

\section{Research on Characteristics of Heavy-load Lifting Machinery Based on Hydraulic-gas Energy Storage and Closed Displacement Controlled System}

\section{HAO Yunxiao XIA Lianpeng QUAN Long ZHAO Bin CHENG Hang XIONG Xiaoyan}

(Key Lab of Advanced Transducers and Intelligent Control System of Ministry of Education and Shanxi Province, Taiyuan University of Technology, Taiyuan 030024)

\begin{abstract}
When the heavy-load lifting machinery using variable speed displacement controlled cylinder system moves downwards, if there are no braking resistors or energy recovery device, the system will not be controlled due to that the electrical motor can't sustain reverse load. However, the braking resistors consumes the generation energy of electrical motor, decreases the energy efficiency of the system and increases the heat dispassion burden. In order to solve the above problems, a closed displacement controlled three-chamber cylinder system of heavy-load lifting system based on hydraulic-gas energy storage is proposed. In the system, hydraulic pump is connected with the rod-less chamber and rod chamber of the three-chamber cylinder to control the lifting machinery to move. The weight balancing chamber of the three-chamber cylinder connected with an accumulator balances the weight of the lifting machinery to directly recycle the potential energy, which can improve the energy efficiency and the operation performance of the system. To research the working performance and energy efficiency of the system, it is used to control the boom of a hydraulic excavator. The parameters of the three-chamber cylinder and the accumulator is determined firstly and then the control strategy of the proposed system is designed. Finally, the test platform of the hydraulic excavator boom controlled by the proposed system is built and the system feasibility is validated. The testing results show that appropriate accumulator pressure not only can realize the boom potential energy recycling to improve the system energy efficie ncy, but also can keep the electrical motor working in motoring condition to improve the system controllability. Compared with the three-chamber cylinder controlled by the load-sensing system, the output energy of the hydraulic pump in the proposed system decreases by $27.2 \%$.
\end{abstract}

Key words: hydraulic excavator; hydraulic-gas energy storage driving; three-chamber cylinder; closed displacement control system; energy recycling

* 国家自然科学基金(U1510206，51675364，51605322)和山西省重点研发 计划(201803D421040)资助项目。20180514 收到调稿, 20190326 收到修 改稿 


\section{0 前言}

与阀控系统相比, 闭式原控系统主回路不需要 控制阀, 不存在节流损失, 油液发热量小, 是实现 工程机械节能减排和绿色发展的有效途径之一 ${ }^{[1]}$ 。 同时, 电驱动力源可避免柴油发动机对环境造成的 污染，也是工程机械的发展方向之一 ${ }^{[2]}$ 。

泵控缸系统分为泵控对称䍂 ${ }^{[3]}$ 和泵控非对称缸 两种, 泵控对称缸技术已较为成熟。泵控非对称缸系 统中, 非对称液压缸两个容腔存在面积差, 需要实时 平衡不对称流量 ${ }^{[4]}$ 。LODEWYKS 采用两种方法解决 泵控非对称缸的不对称流量问题, 一种是采用液压变 压器, 调节液压变压器的配流窗口位置; 另一种是采 用通轴驱动的两个变排量液压洜, 调节变排量液压泵 斜盘摆角 ${ }^{[5]}$ 。陈明东等 ${ }^{[6-7]}$ 对电机驱动两个定量液压䈋 的变转速驱动方案进行研究, 定排量液压泵的排量比 与液压缸两腔面积比相匹配, 通过调节两个定量液压 泵的转速, 控制液压缸运行。张晓刚等 ${ }^{[8-9]}$ 提出一种 三配油窗口的非对称轴向柱塞泵原理; $\mathrm{GE}$ 等 ${ }^{[10-11]}$ 使 用非对称柱塞泵控制挖掘机动臂和斗杆, 获得了良好 的控制特性和节能效果。普渡大学的 BUSQUETS 等 [12-14] 深入研究液控单向阀补油平衡不对称流量的泵 控系统, 并构建全泵控系统混合动力液压挖掘机, 与 负载敏感系统相比，降低能耗达 50\% ${ }^{[15-17]}$, 有效降低 系统装机功率和燃油消耗 ${ }^{[18-19] 。}$

综上所述, 泵控非对称缸系统的流量不对称问 题已有较好的解决方案, 并取得良好的效果, 并可 实现能量的回收和利用。当重载举升机构下降时, 液压缸超越收回, 液压泵将处于液压马达工况, 需 要外界提供能量实时平衡液压马达转矩。对于发动 机驱动的多个泵控系统, 由于各执行器运行工况不 同, 当某个执行器的液压泵处于液压马达工况时, 液压马达转矩将被发动机或其他执行器的液压洜平 衡 ${ }^{[17-20]}$ 。对于变转速闭式葲控系统, 液压缸处于超 越收回工况时，电机将处于发电状态，可采用制动 电阻、电能回馈电网技术或超级电容平衡电机的发 电能量, 进而平衡液压马达的转矩。但电机的发电 能量将被制动电阻转换为热能耗散掉, 降低系统能 量效率, 增加系统的散热负担。对于变转速非对称 泵控系统, 非对称泵的一个油口与蓄能器连接, 当 液压缸超越收回时, 非对称葲向蓄能器充油, 蓄能 器回收能量, 使非对称泵始终处于液压泵工况, 实 现能量平衡 ${ }^{[11]}$ 。

为此, 提出一种基于液气储能的重载举升机构 闭式洜控三腔液压缸系统, 并将其应用于液压挖掘
机动臂。液压泵与三腔液压缸的无杆腔和有杆腔连 接，控制重载举升机构运行，消除系统的节流损失。 三腔液压缸的配重腔与蓄能器连接, 平衡举升机构 的重量 ${ }^{[21]}$, 直接回收利用重载举升机构的势能。通 过合理设置蓄能器压力, 使三腔液压缸始终处于阻 力伸出或阻力收回工况, 提高闭式泵控系统的控制 性能和能量效率。

研究中, 首先对闭式原控三腔液压缸系统的原 理进行分析, 设计三腔液压缸和蓄能器的参数, 制 定闭式泵控三腔液压缸系统的控制策略, 最后构建 闭式原控三腔液压缸的挖掘机动臂试验系统，验证 所提系统的可行性。

\section{1 工作原理}

图 1 所示为提出的基于液气储能的重载举升机 构闭式洜控三腔液压缸系统原理，该系统包括控制 器、伺服电机及驱动器、定排量液压泵、三腔液压 缸、蓄能器、补油系统、液控方向阀及安全阀等。 三腔液压缸包括无杆腔 $\mathrm{A}$ 、有杆腔 $\mathrm{B}$ 和配重腔 $\mathrm{C}$, 配重腔与蓄能器连接, 平衡举升机构自重, 直接回 收利用举升机构重力势能。通过合理设置蓄能器压 力, 可使液压缸在举升机构上升时处于阻力伸出工 况，下降时处于阻力收回工况，防止伺服电机处于 发电工况，提高洜控系统的可控性。采用伺服电机 驱动定排量液压洜，定排量液压泵的两个油口分别 与三腔液压缸的无杆腔和有杆腔连接, 通过改变伺 服电机转速和方向便可控制三腔液压缸的运行速度 和位移。此外, 设置小流量的补油系统, 补偿三腔 液压缸的不对称流量和系统泄漏, 并对三腔液压缸 进行预压紧。

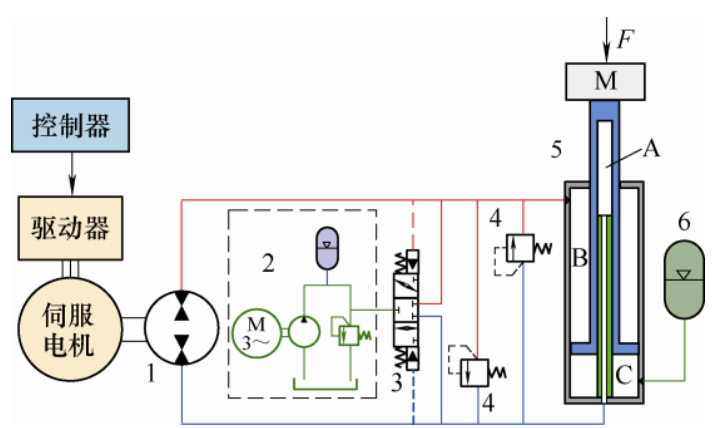

图 1 变转速泵控三腔液压缸原理图

1. 定排量液压泵 2. 补油系统 3. 液控方向阀

4. 安全阀 5. 三腔液压畋 6 . 蓄能器

\section{2 参数设计}

图 2 所示为闭式原控三腔液压缸系统应用于挖 
掘机动臂的原理图。三腔液压缸主要由缸体、活塞 杆以及固定柱塞等组成。挖掘机作业中, 三腔液压 缸的结构尺寸应满足挖掘机的工作要求和工作强 度, 同时蓄能器参数对洜控系统的运行特性会产生 较大影响, 因此, 对三腔液压缸的结构参数和蓄能 器参数进行了分析设计。

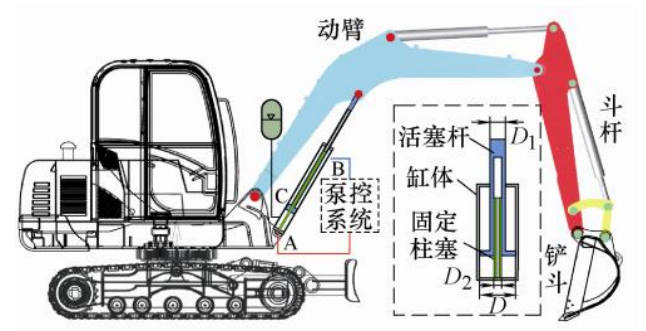

图 2 闭式泵控三腔液压缸驱动挖掘机动臂系统

由于安装空间的限制, 三腔液压缸的外径不能 超过 $140 \mathrm{~mm}$, 为满足结构强度要求, 三腔液压缸的 缸体和活塞杆中空部分的壁厚需要大于 $15 \mathrm{~mm}$ 。依 据原双腔液压缸结构参数, 将三腔液压缸的内径设 计为 $110 \mathrm{~mm}$ 。为保证无杆腔的压力在铲斗满载上升 时不超过系统的额定压力, 并考虑活塞杆中空部分 与固定柱塞之间密封元件的安装, 无杆腔的直径确 定为 $50 \mathrm{~mm}$ 。为保证挖掘机在挖掘作业过程中, 动 臂提供足够的挖掘力, 依据原双腔液压缸的尺寸和 活塞杆强度, 活塞杆直径确定为 $90 \mathrm{~mm}$ 。表 1 所示 为三腔液压缸和原双腔液压缸的结构参数。

表 1 液压缸尺寸

$\mathrm{mm}$

缸体内径 $D$ 活塞杆直径 $D_{1}$ 固定活塞直径 $D_{2}$

\begin{tabular}{cccc}
\hline 三腔液压缸 & 110 & 90 & 50 \\
双腔液压缸 & 100 & 70 & \\
\hline
\end{tabular}

图 3 所示为采用双腔液压缸, 动臂运行过程中 无杆腔的压力测试曲线, 无杆腔压力在动臂下降时 约为 $7 \mathrm{MPa}$, 在动臂上升时约为 $9 \mathrm{MPa}$ 。

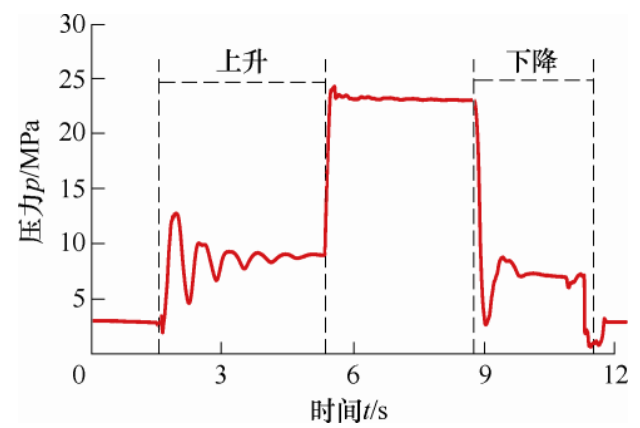

图 3 动臂双腔液压缸压力

动臂动作时间较短, 蓄能器内气体变化可视为 绝热过程, 蓄能器气体状态方程为

$$
p_{\mathrm{x}} V_{\mathrm{x}}^{n}=p_{0} V_{0}^{n}
$$

式中, $p_{\mathrm{x}} 、 V_{\mathrm{x}}$ 为蓄能器气体压力、体积; $p_{0} 、 V_{0}$ 为 蓄能器初始气体压力、体积; $n$ 为气体指数, $n=1.4$ 。

在洜控三腔液压缸系统中, 为充分回收动臂势 能, 并使动臂在无操作下平稳驻停, 根据表 1 中三 腔液压缸与双腔液压缸结构参数, 蓄能器的最高工 作压力 $p_{1}$ 设置为 $12 \mathrm{MPa}$ 。动臂在下降过程中, 蓄 能器最低工作压力不宜过低, 设置蓄能器最低工作 压力 $p_{2}$ 为 $8 \mathrm{MPa}$ 。动臂从最高点下降到铲斗与地面 接触时, 蓄能器的容积变化约为 $2.3 \mathrm{~L}$ 。由于 $0.25 p_{2}<p_{0}<0.9 p_{1}$, 选择蓄能器的充气压力为 5.5 $\mathrm{MPa}$, 由式(1)推导得蓄能器公称容积为 $20 \mathrm{~L}$ 。

\section{3 控制策略}

伺服电机驱动定排量洜，控制三腔液压缸的无 杆腔和有杆腔, 是典型的泵控非对称缸系统, 需要 根据泵控系统的特性制定所提系统的控制策略。

动臂上升和动臂下降时, 闭式洜控三腔液压缸 系统的四象限运行原理如图 4 所示。动臂上升时, 洜控系统将工作在第一象限或第二象限。第一象限 时, 三腔液压缸阻力伸出, 无杆腔为高压, 有杆腔 为低压, 此时无杆腔为控制腔, 由于有杆腔面积大, 有杆腔向补油系统排油。第二象限时, 三腔液压缸 超越伸出, 有杆腔为高压, 无杆腔为低压, 此时有 杆腔为控制腔, 补油系统向无杆腔补油。动臂下降 时, 泵控系统将工作在第三象限或第四象限。第三 象限时, 三腔液压缸阻力收回, 液压泵输出高压油 到三腔液压缸有杆腔, 此时有杆腔为控制腔, 补油 系统向无杆腔补油。第四象限时, 三腔液压缸超越 收回, 无杆腔为高压, 有杆腔为低压, 此时无杆腔 为控制腔, 有杆腔向补油系统排油。

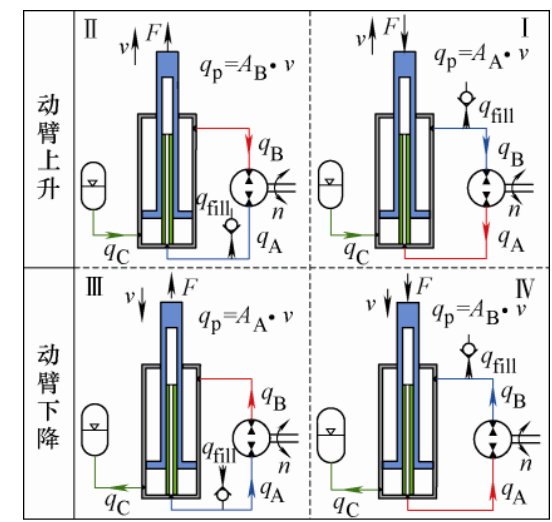

图 4 变转速泵控三腔液压缸的四象限原理

挖掘机大多通过操作人员观察执行机构的位置 和速度, 操纵手柄输出信号, 控制执行器的速度和 位移。手柄操纵时, 产生控制信号 $z$, 对应的三腔 
液压缸活塞杆速度为 $v$, 两者之间的关系为

$$
v=k_{\mathrm{c}} z
$$

式中, $k_{\mathrm{c}}$ 为三腔液压缸速度与手柄信号的比例增益。

忽略三腔液压缸泄漏及油液压缩性, 三腔液压 缸 $A$ 腔和 $B$ 腔的流量方程为

$$
\begin{aligned}
& q_{\mathrm{A}}=A_{\mathrm{A}} v \\
& q_{\mathrm{B}}=A_{\mathrm{B}} v
\end{aligned}
$$

式中, $A_{\mathrm{A}} 、 A_{\mathrm{B}}$ 为三腔液压缸无杆腔和有杆腔的有效 作用面积; $v$ 为三腔液压缸的位移。

忽略液压泵泄漏, 液压泵的流量

$$
q_{\mathrm{p}}=n D_{\mathrm{p}}
$$

式中, $D_{\mathrm{p}}$ 为液压原排量; $n$ 为伺服电机转速。

当洜控系统处于第一象限或第三象限时, 三腔 液压缸无杆腔为控制腔, 伺服电机转速

$$
n=\frac{A_{\mathrm{A}} v}{D_{\mathrm{p}}}
$$

当泵控系统处于第二象限或第四象限时, 三腔
液压缸有杆腔为控制腔，伺服电机转速

$$
n=\frac{A_{\mathrm{B}} v}{D_{\mathrm{p}}}
$$

假设伺服电机的转速与控制信号 $u$ 的关系为

$$
n=k u
$$

式中, $k$ 为伺服电机转速对控制信号的增益。

联立式(2)、(6)、(7)和(8)可得手柄信号与伺服 电机控制信号的关系为

$$
\begin{aligned}
& u=\frac{A_{\mathrm{A}} k_{\mathrm{c}} z}{D_{\mathrm{p}} k} \quad \text { 第一或第三象限 } \\
& u=\frac{A_{\mathrm{B}} k_{\mathrm{c}} z}{D_{\mathrm{p}} k} \quad \text { 第二或第四象限 }
\end{aligned}
$$

图 5 为闭式原控动臂三腔液压缸系统控制策略 原理图。操作手柄输出控制信号, 通过比较三腔液 压缸无杆腔和有杆腔压力，手柄信号将转换为不同 的伺服电机控制信号，从而保证三腔液压缸速度的 平稳性。

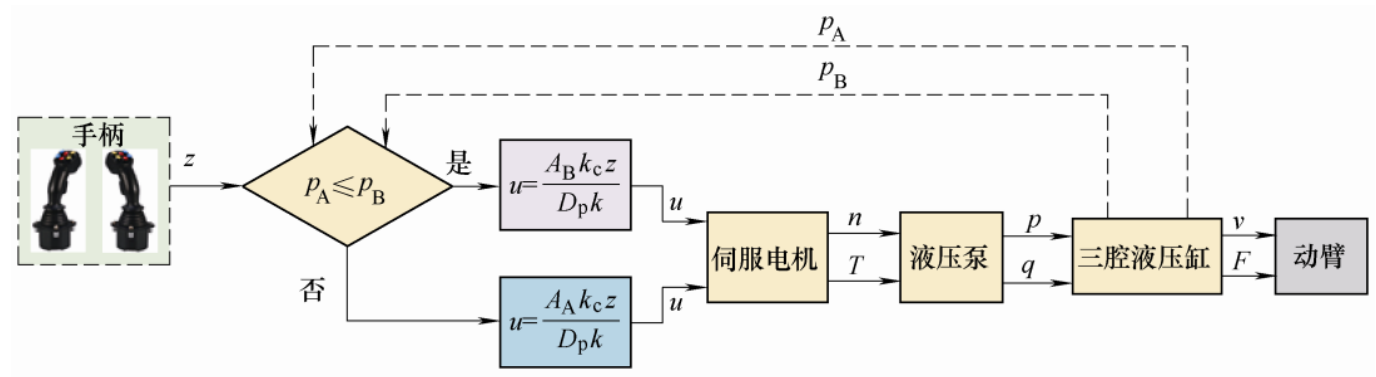

图 5 不控三腔液压缸系统控制策略

\section{4 试验研究}

\section{1 试验系统}

为分析研究重载举升机构闭式泵控三腔液压 缸系统的运行特性和能耗特性, 对实验室 $6 \mathrm{t}$ 液压 挖掘机进行改造, 建立挖掘机动臂闭式洜控三腔
液压缸系统, 试验原理如图 6 所示。试验系统中, dSPACE 输出控制信号到驱动器控制伺服电机, 并 采集手柄信号、三腔液压缸各腔压力、速度和位 移以及伺服电机的转速等信号, 伺服电机驱动定 排量液压泵控制动臂三腔液压缸运行，并外接制 动电阻吸收未被蓄能器回收的挖掘机执行机构重 力势能。
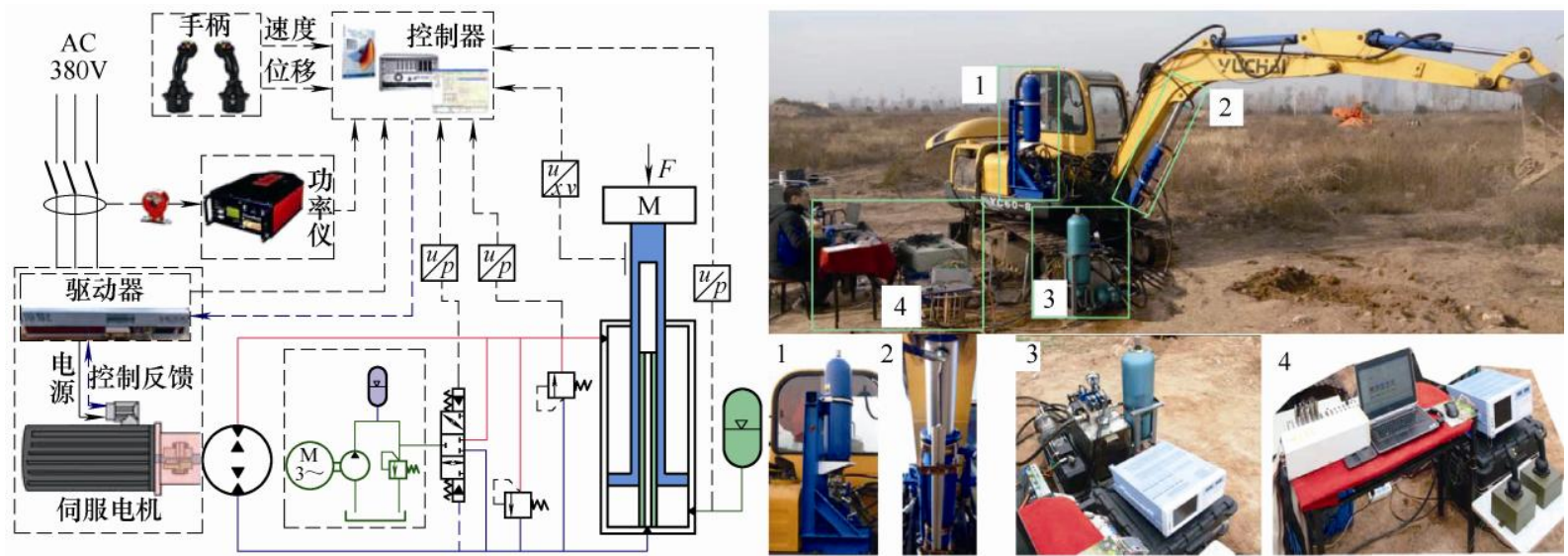

图 6 动臂三腔液压缸闭式㬌控系统试验原理及试验照片

1. 蓄能器 2. 三腔液压缸 3. 原控系统 4. 控制系统 


\section{2 试验结果}

将蓄能器最低工作压力设置为 $8 \mathrm{MPa}$, 操作手 柄控制动臂上升和下降一个周期。图 7 所示为三腔 液压缸速度及压力变化曲线。试验中, $1 \sim 6 \mathrm{~s}$, 手 柄输出正向控制信号, 三腔液压缸伸出, 动臂上升, 三腔液压缸的平均速度约为 $80 \mathrm{~mm} / \mathrm{s}$, 速度平稳。 此时, 三腔液压缸无杆腔压力明显大于有杆腔压力, 无杆腔为液压泵的控制腔。由于配重腔面积比无杆 腔面积大, 配重腔压力减小将使无杆腔压力持续快 速升高, 增大蓄能器体积或压力可减小无杆腔压力, 但会使动臂处于过平衡状态。在动臂上升过程中, 有杆腔持续向补油系统排油, 压力约为 $2.3 \mathrm{MPa}$ 。 $~$ $13 \mathrm{~s}$, 手柄输出负向控制, 三腔液压缸缩回, 动臂 下降, 三腔液压缸速度约为 $100 \mathrm{~mm} / \mathrm{s}$, 速度存在较 小超调, 但速度整体平稳。此时, 三腔液压缸有杆 腔压力明显大于无杆腔压力, 有杆腔为液压泵的控 制腔。在挖掘机执行机构重力和三腔液压缸有杆腔 压力作用下, 三腔液压缸配重腔向储能蓄能器充液, 配重腔压力逐渐升高, 随着配重腔压力的升高, 有 杆腔压力也逐渐升高。由于三腔液压缸有杆腔压力 大于无杆腔压力, 补油系统向无杆腔充油, 无杆腔 压力在 $2.3 \mathrm{MPa}$ 附近波动。

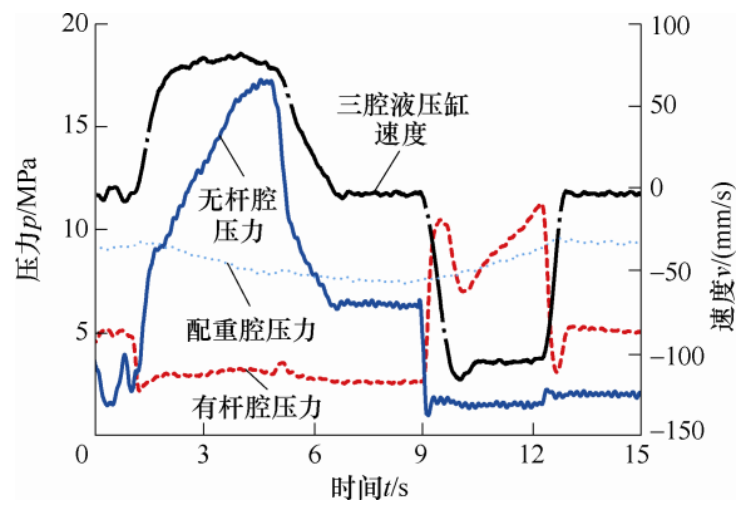

图 7 三腔液压缸速度和压力曲线

液压泵和蓄能器的功率和能量曲线如图 8 所 示。液压洜输出功率与流量和压力有关, 图 8 中可 以看出, 动臂上升时的液压洜输出功率与无杆腔压 力变化规律相同, 动臂下降时的液压洜输出功率与 有杆腔压力变化规律相同, 液压泵峰值功率在动臂 下降过程中, 约为 $3.6 \mathrm{~kW}$ 。由于动臂上升时的速度 较动臂下降时的速度小, 蓄能器充液功率小于蓄能 器放液功率。动臂上升时, 液压洜输出能量约为 6.7 $\mathrm{kJ}$, 下降时约为 $8.5 \mathrm{~kJ}$ 。由于动臂上升和下降距离相 同, 蓄能器回收和释放的能量基本相同, 约为 20.85 $\mathrm{kJ}$ 。假设动臂下降过程的液压泵输出能量均被蓄能 器回收, 则蓄能器回收的动臂势能为 $12.35 \mathrm{~kJ}$ 。

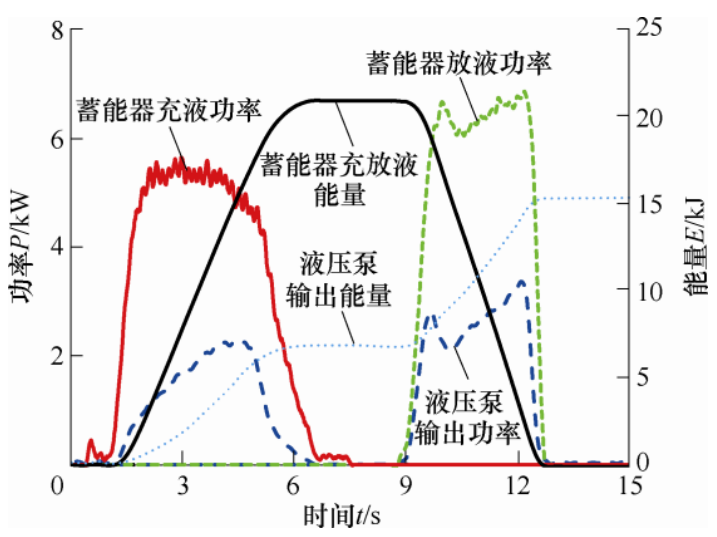

图 8 液压百和蓄能器的功率和能量曲线

闭式洜控系统的能量损失主要是由三腔液压缸 有杆腔向补油系统排油造成的。动臂上升时, 泵控 系统工作在第一象限, 三腔液压缸有杆腔向补油系 统排油, 能量损失约为 $1.12 \mathrm{~kJ}$ 。动臂下降初始阶段, 由图 7 可以看出, 三腔液压缸的无杆腔压力比有杆 腔压力大, 泵控系统短时工作在第四象限, 能量损 失约为 $0.05 \mathrm{~kJ}$ 。当蓄能器最低工作压力为 $8 \mathrm{MPa}$ 时, 动臂下降时, 伺服电机可能短时处于发电状态, 但 发电功率很小, 可被驱动器内部的电容吸收, 为提 高原控系统的午余度, 可附加小功率的制动电阻。

为分析蓄能器压力对系统特性的影响, 设置蓄 能器最低工作压力分别为 $7 \mathrm{MPa} 、 8 \mathrm{MPa}$ 和 $10 \mathrm{MPa}$, 伺服电机的转速控制信号如图 9 所示。

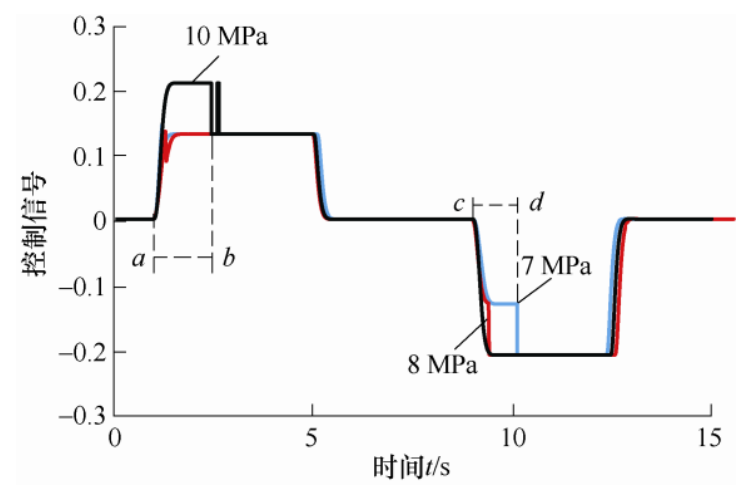

图 9 伺服电机转速控制信号

当蓄能器最低工作压力为 $10 \mathrm{MPa}$ 时, 三腔液 压缸配重腔的支撑力已超过了挖掘机执行机构的重 力, 在动臂上升 $a b$ 段, 三腔液压缸超越伸出, 有杆 腔为控制腔, 泵控系统工作在第二象限, 蓄能器释 放的能量部分被制动电阻消耗。当蓄能器最低工作 压力为 $7 \mathrm{MPa}$ 时, 三腔液压缸配重腔的支撑力不能 够平衡挖掘机执行机构的重量, 在动臂下降 $c d$ 段, 三腔液压缸超越缩回, 无杆腔为控制腔, 泵控系统 工作在第四象限, 挖掘机工作装置部分势能被制动 电阻消耗。当蓄能器最低工作压力为 $8 \mathrm{MPa}$ 时, 泵 
控系统在动臂上升时基本处于第一象限, 在动臂下 降时基本处于第三象限。因此, 蓄能器的最低工作 压力为 $8 \mathrm{MPa}$, 可避免动臂运行过程中, 伺服电机 处于发电状态造成的能量浪费, 提高汬控系统的能 量效率和控制性能。

图 10 所示为不同蓄能器最低工作压力的液压 泵输出能量。动臂上升时, 蓄能器压力越高, 液压 原输出能量越少, $7 \mathrm{MPa}$ 时液压泵的输出能量为 $10.1 \mathrm{~kJ}, 10 \mathrm{MPa}$ 时液压洜的输出能量仅为 $1.2 \mathrm{~kJ}$ 。 与动臂上升相反, 蓄能器压力的增加, 将导致液压 泵在动臂下降过程输出能量的增加, $7 \mathrm{MPa}$ 时液压 泵的输出能量为 $4.7 \mathrm{~kJ}, 10 \mathrm{MPa}$ 时液压泵输出能量 骤升至 $14.6 \mathrm{~kJ}$ 。综合分析图 9 和图 10 可知, 当蓄 能器的最低工作压力为 $8 \mathrm{MPa}$ 时, 原控系统具有较 好的运行特性和能耗特性。

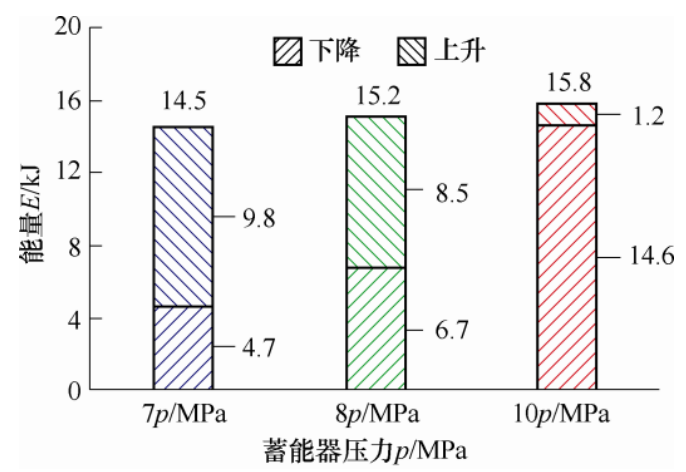

图 10 蓄能器压力不同时, 液压泵输出能量

由前期研究工作 ${ }^{[21]}$ 可知, 与双腔液压缸负载敏 感控制系统相比, 三腔液压缸负载敏感控制系统可 降低能耗 $48.57 \%$ 。图 11 所示为蓄能器最低工作压 力为 $8 \mathrm{MPa}$, 动臂上升和下降一个周期, 负载敏感 控制三腔液压缸系统和闭式泵控三腔液压缸系统的 液压泵输出能量。由于负载敏感系统存在较大节流 损失，负载敏感系统的液压洜输出能量明显高于闭 式洜控系统, 负载敏感系统的液压泵输出能量为 $20.9 \mathrm{~kJ}$ 。闭式泵控系统的液压洜输出能量为 $15.2 \mathrm{~kJ}$, 闭式洜控系统可进一步节能约 $27.2 \%$ 。

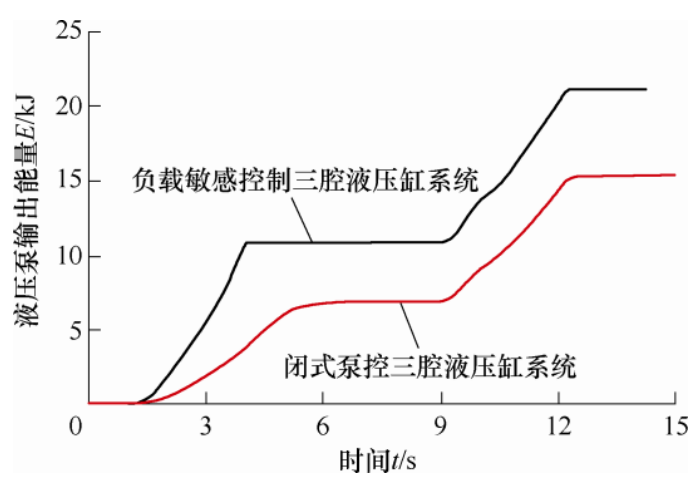

图 11 负载敏感系统和泵控系统的液压泵输出能量

\section{5 结论}

（1）提出一种重载举升机构的液气储能闭式洜 控三腔液压缸系统原理, 三腔液压缸的配重腔与蓄 能器连接直接回收利用举升机构势能, 平衡举升机 构重量。泵控系统只需要输出较小能量驱动负载, 并且消除系统的节流损失，提高系统的能量效率。

(2) 试验结果表明, 蓄能器压力将影响闭式原 控三腔液压缸系统运行特性和能耗特性, 综合考虑, 当蓄能器最低压力为 $8 \mathrm{MPa}$ 时, 所提系统的运行特 性和能效特性俱佳，与负载敏感控制三腔液压缸系 统相比，闭式原控系统可进一步节能 $27.2 \%$ 。

\section{参 考 文 献}

[1] QUAN Zongyi， QUAN Long, ZHANG Jinman. Review of energy efficient direct pump controlled cylinder electro-hydraulic technology[J]. Renewable and Sustainable Energy Reviews, 2014(35): 336-346.

[2] LAJUNEN A, SAINIO P, LAURILA L, et al. Overview of powertrain electrification and future scenarios for non-road mobile machinery[J]. Energies, 2018, 11(5): 1184

[3] HABIBI S, GOLDENBERG A. Design of a new high performance electro hydraulic actuator[C]IUIEEE/ASME International Conference on Advanced Intelligent Mechatronics, Atlanta, USA, 19-23 September, 1999: 227-232.

[4] 权龙. 原控缸电液技术研究现状、存在问题及创新解决 方案[J]. 机械工程学报，2008，44(11)：87-92.

QUAN Long. Current state, problems and the innovative solution of electro-hydraulic technology of pump controlled cylinder[J]. Chinese Journal of Mechanical Engineering, 2008, 44(11): 87-92.

[5] LODEWYKS J. Varible displacement pump closed loop controlled differential cylinder system[D]. Aachen : RWTH, 1994.

[6] 陈明东, 赵丁选, 倪涛. 液压挖掘机动臂闭式油路节能 系统[J]. 吉林大学学报, 2012, 42(5): 1140-1144.

CHEN Mingdong, ZHAO Dingxuan, NI Tao. Boom energy saving system with closed circuit in hydraulic excavator[J]. Journal of Jilin University, 2012，42(5): $1140-1144$

[7] CHEN Mingdong, ZHAO Dingxuan. The gravitational potential energy regeneration system with closed-circuit of boom of hydraulic excavator[J]. Mechanical Systems \& Signal Processing, 2017(82): 178-192.

[8] 张晓刚，权龙，杨阳，等. 并联型三配流窗口轴向柱塞 
葲特性理论分析及试验研究[J]. 机械工程学报, 2011, 47(14): 151-157.

ZHANG Xiaogang, QUAN Long, YANG Yang, et al. Theoretical analysis and experimental research on characteristics of parallel three assignment windows axial piston pump[J]. Journal of Mechanical Engineering, 2011, 47(14): 151-157.

[9] HUANG Jiahai, QUAN Long, ZHANG Xiaogang. Development of a dual-acting axial piston pump for displacement-controlled system[J]. Proceedings of the Institution of Mechanical Engineers Part B Journal of Engineering Manufacture, 2014, 228(4): 606-616.

[10] GE Lei, QUAN Long, LI Yunwei, et al. A novel hydraulic excavator boom driving system with high efficiency and potential energy regeneration capability[J]. Energy Conversion and Management, 2018(166): 308-317.

[11] 葛否, 张晓刚, 权龙, 等. 变转速非对称泵直驱液压挖 掘机斗杆试验研究[J]. 机械工程学报, 2017, 53(16):

210-216.

GE Lei, ZHANG Xiaogang, QUAN Long, et al. Experiment study on the characteristics of speed-variable asymmetric pump driven excavator arm[J]. Journal of Mechanical Engineering, 2017, 53(16): 210-216.

[12] BUSQUETS E, IVANTYSYNOVA M. A multi-actuator displacement- controlled system with pump switching: A study of the architecture and actuator-level control[J]. International Journal of Fluid Power System, 2015(8): 66-75.

[13] BUSQUETS E, IVANTYSYNOVA M. Discontinuous projection-based adaptive robust control for displacement-controlled actuators[J]. Journal of Dynamic Systems Measurement \& Control, 2015, 137(8): 1-10.

[14] RAHMFELD R, IVANTYSYNOVA M. Displacement controlled linear actuator with differential cylinder: A way to save primary energy in mobile machines[C]//Fifth International Conference on Fluid Power Transmission and Control. Hangzhou, China, 3-6 April, 2001 : 296-301.

[15] ZIMMERMAN J, IVANTYSYNOVA M. The effect of system pressure level on the energy consumption of displacement controlled actuator systems[C]//Proceedings of the 5th FPNI PhD Symposium. Krakow, Poland, 1-5 July, 2008. 2008: 77-92.
[16] ZIMMERMAN J , IVANTYSYNOVA M. Effect of installed hydraulic corner power on the energy consumption and performance of multi-actuator displacement controlled mobile machines[C]//ASME 2009 Dynamic Systems and Control Conference. Ann Arbor, Michigan, USA, 20-22 October, 2009: 871-878.

[17] HIPPALGAONKAR R, IVANTYSYNOVA M. A series-parallel hydraulic hybrid mini-excavator with displacement controlled actuators $[\mathrm{C}] / /$ Scandinavian International Conference on Fluid Power, Linköping, Sweden, 3-5 June, 2013: 31-42.

[18] HIPPALGAONKAR R, IVANTYSYNOVA M. Optimal power management of hydraulic hybrid mobile machines-part I: theoretical studies, modeling and simulation[J]. Journal of Dynamic Systems Measurement \& Control, 2016, 138(5): 1-12.

[19] HIPPALGAONKAR R, IVANTYSYNOVA M. Optimal power management of hydraulic hybrid mobile machines-Part II : machine implementation and measurements[J]. Journal of Dynamic Systems Measurement \& Control, 2016, 138(5): 1-10.

[20] 赵鹏宇, 陈英龙, 周华, 等. 油液混合动力挖掘机势能 回收及能量管理策略 $[\mathrm{J}]$. 浙江大学学报, 2016, 50(5): 893-901.

ZHAO Pengyu, CHEN Yinglong, ZHOU Hua, et al. Potential energy recovery management strategy of hydraulic hybrid excavator[J]. Journal of Zhejiang University, 2016, 50(5): 893-901.

[21] 夏连鹏, 权龙, 张晓刚, 等. 液气复合驱动液压挖掘机 动臂运行特性及能效 [J]. 机械工程学报, 2017, 53(20): 176-183.

XIA Lianpeng, QUAN Long, ZHANG Xiaogang, et al. Operating characteristics and energy efficiency of hydraulic-gas combined driving hydraulic excavator boom[J]. Journal of Mechanical Engineering, 2017, 53(20): 176-183.

作者简介: 郝云晓, 男, 1990 年出生, 博士研究生。主要研究方向为流 体传动与控制、机电装备的电液智能与节能控制。

E-mail: haoyunxiaosuper@163.com

权龙(通信作者), 男, 1959 年出生, 博士, 教授, 博士研究生导师。主 要研究方向为电液伺服及比例控制技术。

E-mail: quanlong@tyut.edu.cn 